\title{
3iffusion tensor imaging to visualize axons in the setting of nerve injury and recovery
}

\author{
Thomas Anthony Gallagher, MD,, Neil G. Simon, PhD, FRACP,, ${ }^{3,4}$ and Michel Kliot, MD² \\ Departments of ${ }^{1}$ Radiology and ${ }^{2}$ Neurosurgery, Northwestern University Feinberg School of Medicine, Chicago, Illinois; ${ }^{3}$ Prince \\ of Wales Clinical School, University of New South Wales, Sydney; and 4Department of Neurology, St. Vincent's Hospital, \\ Darlinghurst, New South Wales, Australia
}

\begin{abstract}
Successful management of peripheral nerve trauma relies on accurate localization of the injury and grading of the severity of nerve injury to determine whether surgical intervention is required. Existing techniques, such as electrodiagnostic studies and conventional imaging modalities, provide important information, but are limited by being unable to distinguish severe nerve lesions in continuity that will recover from those that will not. Diffusion tensor imaging (DTI) and tractography of peripheral nerves provide a novel technique to localize and grade nerve injury, by assessing the integrity of the nerve fibers across the site of nerve injury. Diffusion tensor imaging and tractography also hold promise as markers of early nerve regeneration, prior to clinical and electrodiagnostic evidence of recovery. In the present review, the techniques of peripheral nerve DTI and tractography are discussed with respect to peripheral nerve trauma, with illustrative cases demonstrating potential roles of these novel approaches.

http://thejns.org/doi/abs/10.3171/2015.6.FOCUS15211
\end{abstract}

KEY WORDS peripheral nerve trauma; diffusion tensor imaging; tractography; MRI; nerve regeneration

$\mathrm{P}$ ERIPHERAL nerve trauma is common, frequently affecting younger people of working age. Neuropraxic nerve injuries may result in initially severe deficits, but produce no lasting functional abnormalities after the provoking insult is eliminated. In patients with axonal injury (with axonotmetic and neurotmetic histopathological changes), outcomes range from complete spontaneous functional recovery with mild axonotmetic nerve injuries, to no spontaneous functional recovery with neurotmetic nerve injuries. It is in the group of patients with axonal injury where appropriate interventions may improve functional outcomes, but selection of appropriate patients is essential to optimize results.

The development of a noninvasive means to identify axonal loss and early regeneration has important implications for the management of patients with peripheral nerve trauma, in particular the early identification of patients with severe nerve lesions that are unlikely to spontaneously regenerate. Such a tool may facilitate early surgical exploration in appropriate patients and avoid unnecessary surgical interventions in others.
This review discusses the current approaches to measuring nerve injury and recovery, the evolution of diffusion tensor imaging (DTI) for the monitoring of nerve degeneration and regeneration, and illustrative clinical applications of DTI in the nerve injury clinic.

\section{Assessments in Nerve Injury Clinical and Electrodiagnostic Approaches}

Current approaches to diagnosing, quantifying, and monitoring peripheral nerve trauma include clinical assessment and electrodiagnostic studies, supplemented by imaging and intraoperative electrophysiological studies in appropriate cases..$^{13}$ These assessment modalities provide invaluable information, but each one has limitations.

Clinical assessment provides information regarding the distribution of the nerve injury, potential contributing mechanisms, and functional status. However, clinical examination is insensitive to early spontaneous recovery, because muscle or limb movement may not be detectable when only a small number of motor units have re-innervat-

ABBREVIATIONS DTI = diffusion tensor imaging; EMG = electromyography; FA = fractional anisotropy; $M R C=$ Medical Research Council; $T A=$ tibialis anterior. SUBMITTED April 29, 2015. ACCEPTED June 16, 2015. 
ed. Secondary changes in joints and ligaments may further confound the clinical picture.

In terms of electrodiagnostic studies, nerve conduction studies provide a means to quantify the extent of nerve injury, although these are insensitive to smaller increments of nerve regeneration or deterioration. Electromyography (EMG) is more sensitive than clinical examination to detect mild muscle denervation ${ }^{10}$ and early muscle re-innervation. Electromyography may also provide an indication of the time course of the nerve injury in partial injuries if this is not clear from the history. However, EMG will not detect nerve regeneration before nerve fibers have reached the target muscle, which may necessitate several months of monitoring before a nerve starts to regenerate and reach the target muscle, particularly in more proximal nerve injuries.

Intraoperative electrophysiological studies may provide important information regarding the severity of nerve injury and the likelihood of spontaneous regeneration and help guide treatment choices, in particular for those patients in whom grafting and other reconstructive procedures are needed. ${ }^{5,7}$ However, these studies are necessarily invasive.

\section{Imaging in the Setting of Nerve Injury and Recovery}

Imaging is frequently performed in the setting of nerve injury. Muscle imaging, including MRI and ultrasound, has a role in the work-up of nerve injuries. ${ }^{12}$ In particular, muscle imaging identifies changes of denervation edema within days, ${ }^{3,15}$ before the appearance of fibrillations on EMG, and hence may be useful in the hyperacute setting to determine the pattern of muscle denervation. However, it has not yet been elucidated whether the presence of acute muscle edema may distinguish between a severe neuropraxic lesion and one involving axonal injury.

Magnetic resonance neurography, which indicates a series of MRI sequences that include a heavily fat-saturated T2-weighted sequence, ${ }^{1}$ may provide information about the location of nerve injury, the anatomical context of the nerve injury, and associated soft-tissue injury. Emerging 3D anatomical sequences, including T2-weighted and STIR SPACE (sampling perfection with applicationoptimized contrasts using varying flip-angle evolutions; Siemens USA), have introduced greater anatomical detail; the 3D-image volume can be rotated and tilted in any orientation while maintaining a high degree of anatomical fidelity. This provides a means for advanced image postprocessing to best profile longer stretches of nerves, which often assume nonorthogonal courses (e.g., brachial plexus). Magnetic resonance neurography can depict T2signal changes in the nerve, which may also evolve with recovery of nerve injury, although resolution of nerve-signal abnormalities may take many months. ${ }^{2}$ However, in severe nerve injuries in continuity, MR neurography is not useful for predicting which nerve lesions will or will not regenerate spontaneously.

Advanced MRI applications, in particular DTI and tractography, may prove valuable in the workup and monitoring of nerve injury. In DTI, a series of diffusionsensitizing gradients from the MRI scanner are applied in numerous (at least 6) noncollinear directions that capture information that fully characterizes a diffusion tensor. The diffusion tensor is a matrix of values that quantifies the amount (eigenvalues $\lambda_{1} \lambda_{2} \lambda_{3}$ ) and principal directions (eigenvectors $\mathbf{e}_{1} \mathbf{e}_{2} \mathbf{e}_{3}$ ) of diffusion in any given voxel in tissue of interest, including a nerve. The magnitude and direction of the largest movement of diffusion are given by $\lambda_{1}$ and $\mathbf{e}_{1}$ respectively.

With these tools, diffusion of water in each voxel can then be modeled and elegantly visualized as a 3D ellipsoid. If there are no barriers to diffusion (e.g., muscle fibers, nerve fascicles), diffusion is assumed to be isotropic, wanders equally in all directions, and the diffusion tensor predicts a perfect $3 \mathrm{D}$ sphere $\left(\lambda_{1}=\lambda_{2}=\lambda_{3}\right)$. Intact myelinated nerves and fascicles tend to constrain and direct diffusion strictly along a dominant, principal pathway (anisotropic diffusion), and the tensor predicts a series of successive, elongated cigar-shaped ellipsoids that serve as a proxy for the delicate microstructure of the nerve itself., ${ }^{4,9}$

Additional metrics that can be derived from the diffusion tensor include axial diffusivity $\left(\lambda_{1}\right.$, along the principal direction) and radial diffusivity $\left(\lambda_{2}+\lambda_{3} \div 2\right.$, perpendicular to the nerve). Another common metric is fractional anisotropy (FA), an index of how different the principal directions of diffusion are, which ranges from 0 to 1 . High FA along the course of a nerve indicates that it is directing diffusion very strictly in 1 direction along its axis, without gaps or defects. For example, intact and healthy nerves should register as cigar-shaped ellipsoids with high FA. Nerve injury, such as injury to myelin and/or axons, is associated with reduced FA (i.e., less anisotropic water diffusion, more spherical ellipsoids) ${ }^{16}$ Fractional anisotropy increases during nerve regeneration. ${ }^{14}$

Diffusion tensor (DT) tractography uses methods that draw directionally color-encoded streamlines that visually link dominant directions of diffusion (eigenvectors) across many voxels in a target tissue. In general, many different mathematical propagation algorithms exist for DT tractography. Most of them usually accept certain user-defined FA thresholds, tract-length constraints, turning-angle constraints, and desired regions of interest before calculating the most likely route through the tissue. If highly reliable diffusion data are acquired, the resultant 3D fiber bundle is a proxy for nerve fascicles. ${ }^{8}$

\section{Clinical Applications}

Diffusion tensor imaging outside of the brain often requires adjustment of MRI parameters to optimize the image acquisition, and exams must be tailored to the region of interest. Diffusion weightings ranging between $b=$ $600 \mathrm{sec} / \mathrm{mm}^{2}$ and $\mathrm{b}=1000 \mathrm{sec} / \mathrm{mm}^{2}$ are commonly used. Several baseline b-zero images (i.e., without diffusion weighting) are acquired and averaged for use in calculating values for the diffusion tensor. The number of diffusion directions applied is typically between 12 and 30 , and increasing averages can improve the signal-to-noise ratio. Careful selection of appropriate MR-imaging coils (essentially, antennas that transmit and/or receive the MRI signals) that house an area of interest is vital. Acquisition of desired anatomical structural imaging (T1 weighted, T2 weighted, STIR, T1 weighted postcontrast) in the same 
plane as the diffusion imaging is useful for subsequent image registration and review.

\section{Example 1: Visualizing Nerve Regeneration}

A 25-year-old man suffered a laceration to his popliteal fossa from a concrete saw (case previously described by Simon et al. ${ }^{11}$ ). This injury resulted in foot drop with Medical Research Council (MRC) Grade 0/5 weakness of ankle dorsiflexion and toe extension, and Grade 4/5 weakness of eversion. Electrodiagnostic studies performed 4 months after the injury demonstrated total denervation of muscles supplied by the deep peroneal nerve (tibialis anterior [TA] and extensor hallucis longus), and only partial denervation changes in muscles supplied by the superficial peroneal nerve. Magnetic resonance neurography demonstrated an enlarged and T2-hyperintense peroneal nerve at the site of the laceration, and denervation edema of the TA and extensor digitorum longus. Tractography demonstrated no nerve fibers distal to the site of laceration (Fig. 1).

Surgical exploration was undertaken 4 months after nerve injury. This identified that the deep branch of the peroneal nerve was severely injured and did not transmit electrical impulses on intraoperative electrophysiological studies. A 7.5-cm segment of fibrosis was resected and the gap interposed with 3 sural nerve grafts.

One month after surgery, clinical and EMG findings were unchanged, but MR tractography identified a few nerve fibers extending into the grafts. Thirteen months after surgery, TA strength was Grade $4+/ 5$ and extensor hallucis longus strength was Grade 3/5. Electromyography demonstrated evidence of re-innervation of these muscles, and MR tractography identified a compact bundle of nerve fibers extending through the grafts.

\section{Example 2.1: Identifying Severe Nerve Injury}

A 59-year-old, right-handed man suffered a glass laceration to his right medial elbow, which was sutured in the emergency room. Six months after the injury, he was referred for further workup due to persistent right-hand weakness. He was unable to voluntarily contract his left ulnar hand intrinsic muscles, such as the dorsal and palmar interossei muscles. However, he was able to flex his wrist in an ulnar direction, as well as flex the distal interphalangeal joint of the fifth digit at a Grade 4/5 motorstrength level. Sensation was markedly diminished in the fourth and fifth digits of his right hand.

Nerve conduction studies and EMG confirmed a severe but partial right ulnar nerve injury. An ultrasound study at the site of his laceration showed what appeared to be a traumatic neuroma involving the right ulnar nerve, which thinned significantly before enlarging more distally, suggesting interruption of nerve continuity. An MR DTI study confirmed that axons within the right ulnar nerve came to an abrupt end at the proximal portion of the neuroma (Fig. 2). At surgery, the right ulnar nerve was exposed and found to end in a neuroma.

Electrical stimulation of the nerve proximal and distal to the neuroma did not give rise to any contraction in right ulnar-supplied muscles in either the forearm or hand. Stimulating the right ulnar nerve just proximal to
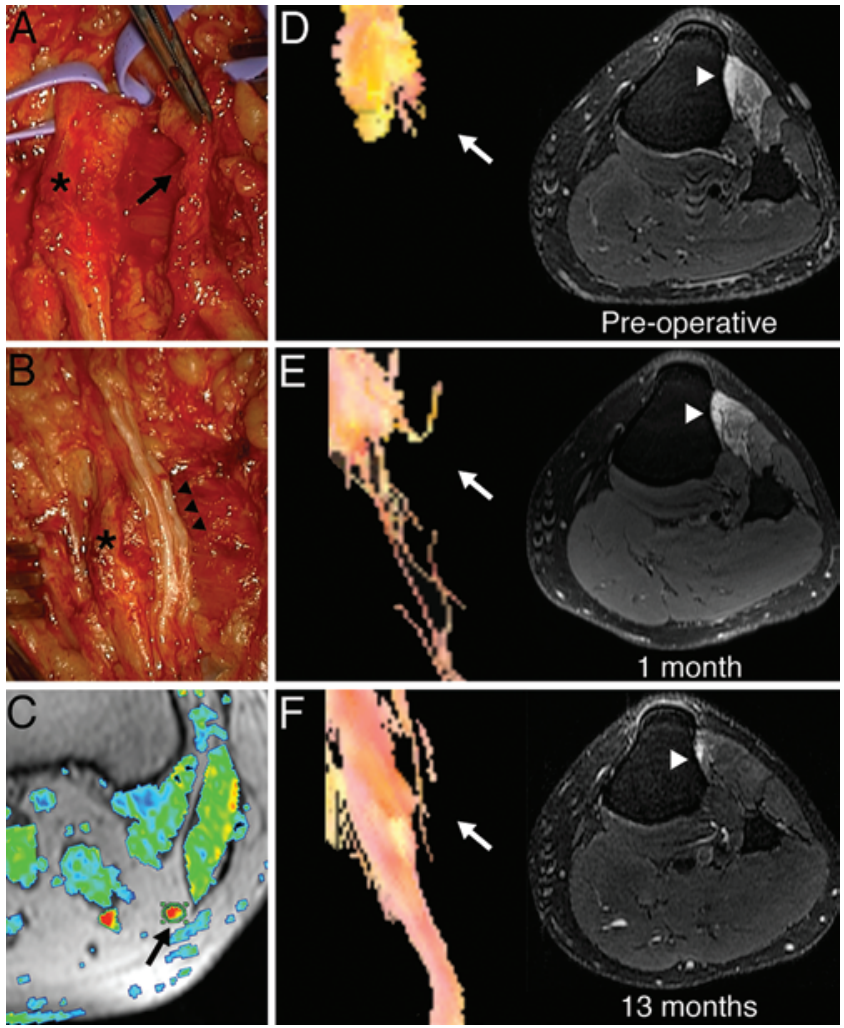

FIG. 1. Operative exploration of the left peroneal nerve identified a damaged, scarred, deep peroneal nerve branch (black arrow, $\mathbf{A}$ ) and relatively preserved superficial peroneal nerve branch (asterisk, $\mathbf{A}$ and $\mathbf{B}$ ). The deep peroneal nerve branch was resected and the resulting defect bridged with 3 sural nerve grafts (black arrowheads, B). A thresholded FA map (C) is shown fused to the corresponding axial T1-weighted image. The proximal common peroneal nerve is identified as a region of increased FA signal (arrow) and is encircled by a region of interest used to calculate average FA values. Preoperative DT tractography demonstrates the proximal stump of the deep peroneal nerve branch, with no axons identified distal to the point of transection (white arrow, D), and T2-weighted, fat-suppressed imaging of the proximal left leg identifies denervation edema in deep peroneal-innervated muscles (TA and extensor digitorum longus, white arrowhead). Repeat DT tractography performed 1 month after surgical repair identifies sparse, disorganized axons distal to the proximal graft neurography (white arrow) and persistent muscle edema (E). Tractography performed 13 months after nerve graft repair demonstrates an organized bundle of fibers traversing the nerve grafts from the proximal graft anastomosis (white arrow) and resolution of muscle edema (F). From Simon et al: Visualizing axon regeneration after peripheral nerve injury with magnetic resonance tractography. Neurology 83:1382-1384, 2014. Copyright Wolters Kluwer Health. Published with permission.

the traumatic neuroma did give rise to a centrally propagating, somatosensory evoked potential response over the contralateral scalp. The 2 findings confirmed the absence of axons distal to the traumatic neuroma, which was also visualized on the preoperative DTI study.

Stimulation of the adjacent median nerve did give rise to contraction in several muscles usually supplied by the ulnar nerve, indicating the presence of a medial-to-ulnar nerve anastomosis (Martin-Gruber) in the forearm, accounting for his ability to flex his wrist in an ulnar direction as well as flex the distal interphalangeal joint of the fifth digit. A 3-cm segment of scarred neuroma tissue was 

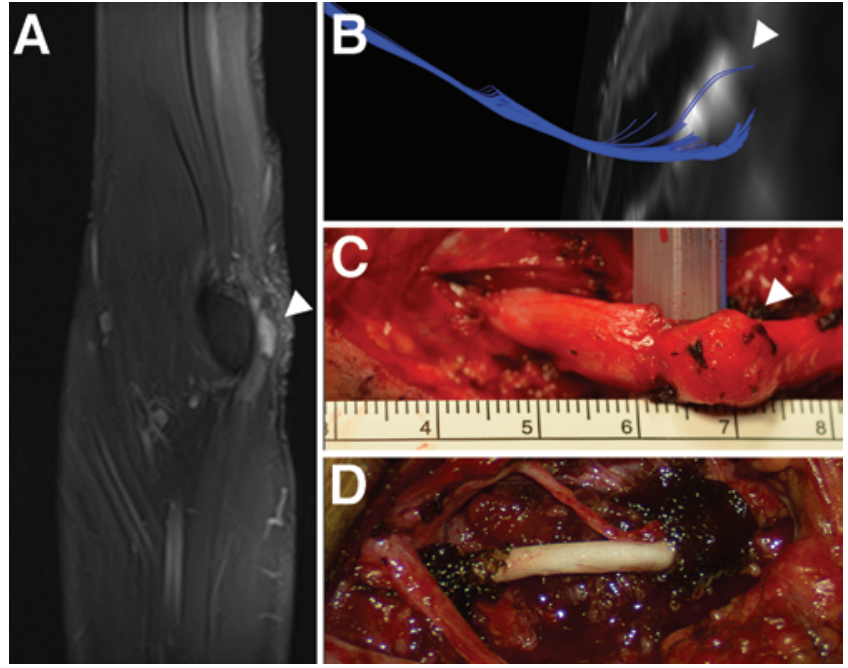

FIG. 2. An ulnar neuroma (arrowhead) was identified on sagittal T2weighted, fat-suppressed MRI as a hyperintense expansion at the level of the medial epicondyle (A). Diffusion tensor tractography (B) of the proximal ulnar nerve demonstrated fibers abruptly ending and fanning around a traumatic neuroma (arrowhead) posterior to the right medial epicondyle (DTI tractography performed using DSI Studio software [dsi-studio.labsolver.org]). The ulnar neuroma (arrowhead) was identified at surgery (C), resected, and the resulting defect bridged with a cadaveric nerve graft (D). Tractography was performed using DSI Studio software. ${ }^{17}$

resected, and 2 cadaveric nerve grafts were interposed between the proximal- and distal-cut ends of the ulnar nerve.

\section{Example 2.2: Identifying Severe Nerve Injury}

A 24-year-old, right-handed man suffered a severe right brachial plexus injury from a motor vehicle accident, resulting in a flail right upper extremity. Emergency surgery was necessary for a vascular repair involving a right subclavian-to-brachial artery bypass with a saphenous vein graft. At the time of this surgery, he was noted to have disruption of multiple nerve elements. A postoperative MRI study visualized complete avulsion of his right C-8 spinal nerve root, partial avulsion of his right $\mathrm{C}-5$ and $\mathrm{C}-6$ spinal nerve roots that appeared to lose continuity more distally at the level of the upper trunk, an injured C-7 spinal nerve root that maintained continuity, and an intact right T-1 spinal nerve root (Fig. 3). At the time of his second surgery 2 months after the accident, his right C-5 and C-6 spinal nerves were found interrupted at the level of the upper trunk, corresponding to what had been visualized on his preoperative MR DTI study. A $2.5-\mathrm{cm}$ cadaveric nerve graft was interposed between the proximal and distal ends of his disrupted upper trunk. A damaged phrenic nerve branch was also neurotized into the distal upper trunk.

\section{Example 3: Localizing Nerve Injury}

A 32-year-old, right-handed woman presented with episodic tingling in her left thumb, index, and middle fingers, which was exacerbated by certain postures and activities. She reported a firm protuberance in the left side of her neck, which was sometimes painful. Strength in her left upper extremity was normal. A radiograph (Fig. 4) showed a large cervical rib complex. The distortion of the normal
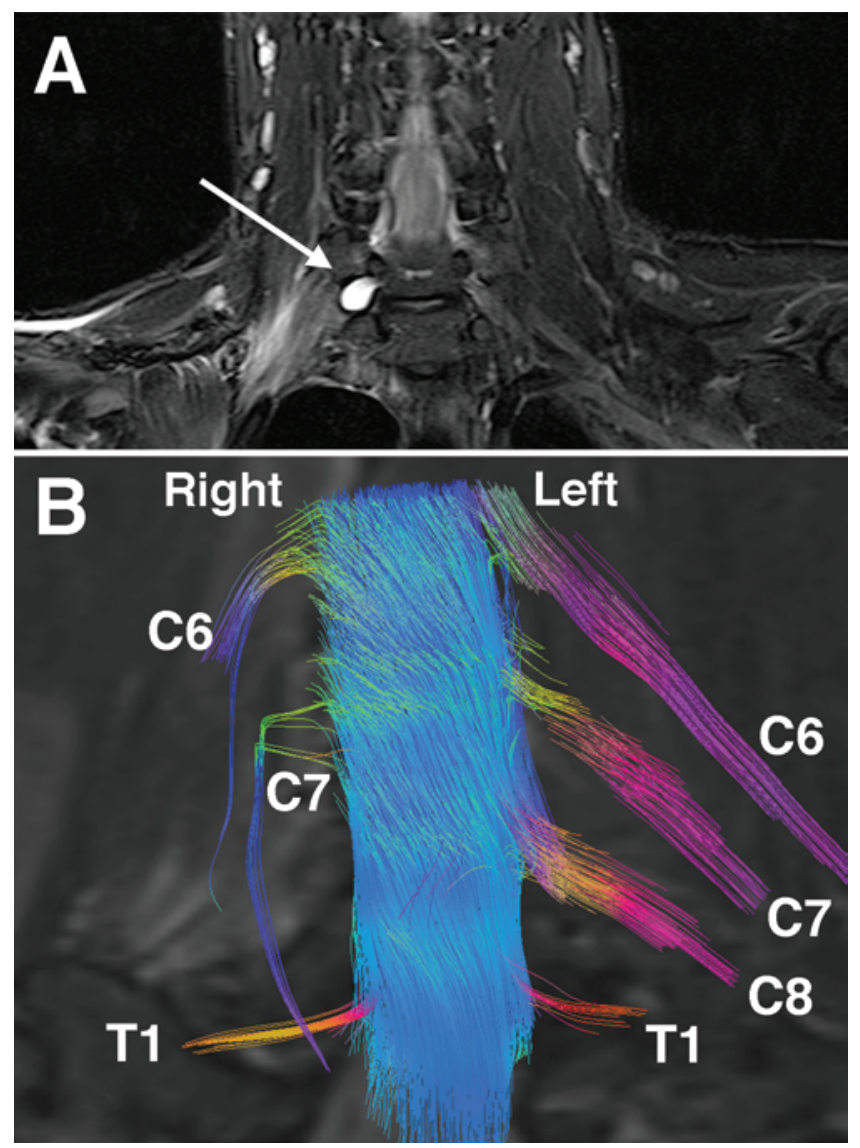

FIG. 3. Coronal STIR image of the cervical nerve roots (A) identified a pseudomeningocele of the right $\mathrm{C}-8$ nerve root (arrow). Diffusion tensor tractography (B) demonstrated reduced nerve fibers in the right C- 6 and C-7 nerve roots and no trackable nerve fibers in the right $\mathrm{C}-8$ nerve root. Tractography was performed using MedINRIA: Medical Image Navigation and Research Tool by INRIA. In Proc. of MICCAI '07 Workshop on Interaction in Medical Image Analysis and Visualization. Brisbane, Australia, 2007.

course of these brachial plexus elements was visualized on an MR DTI study.

\section{Limitations and Future Directions}

Diffusion tensor imaging in peripheral nerves presents several unique challenges, the first of which is the scale of the process we hope to investigate, i.e., diffusion within and alongside very tiny structures. Given the very small size of nerves, the age-old MRI-related struggles among voxel size, spatial resolution, and signal-to-noise ratio have become very relevant in DTI. Reducing the field of view, or focusing in closely to study a single nerve, is not possible in MRI due to a phenomenon called aliasing, or wrap, which literally means that the image wraps on top of itself, obscuring details. Diffusion tensor imaging itself is susceptible to a host of other undesirable factors including patient motion, echo planar distortions, and eddy currents induced by rapidly changing gradients. These distortions introduce noise and spurious effects that impact the sensitive diffusion information we hope to elicit. These can certainly impact the validity of tractography attempts and must be considered. ${ }^{6}$ 

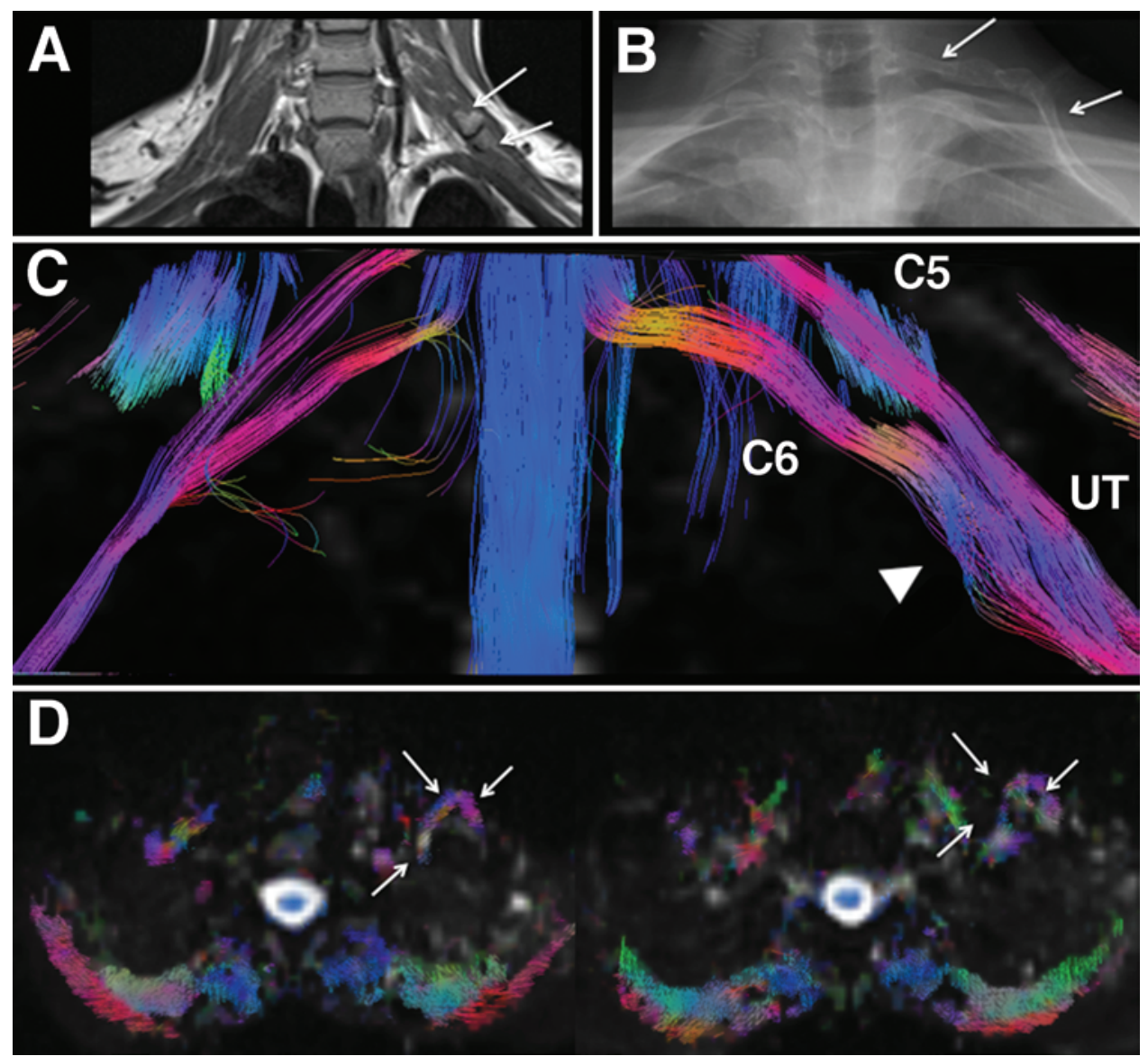

FIG. 4. A coronal T1-weighted spin echo MR image (A) and chest radiograph (B) demonstrated an anomalous cervical rib associated with the left $\mathrm{C}-7$ transverse process (white arrows). Diffusion tensor tractography (C and D) demonstrated that the left $\mathrm{C}-5$ and C-6 nerve roots and upper trunk (UT) (arrowhead [in C] and white arrows [in D]) were splayed around the cervical rib. Tractography was performed using MedINRIA: Medical Image Navigation and Research Tool by INRIA. In Proc. of MICCAI '07 Workshop on Interaction in Medical Image Analysis and Visualization. Brisbane, Australia, 2007.

Fortunately, exciting new MRI sequences are becoming available for clinical use and include a segmented readout of echo trains for diffusion imaging, which reduces echo times, reduces echo planar image distortions, and greatly improves image quality (e.g., RESOLVE [readout segmentation of long variable echo trains], Siemens Medical Solutions USA). Reduced field-of-view techniques (e.g., FOCUS, GE; ZOOMit, Siemens Medical Solutions USA) have been developed that allow selective excitation, allowing a sought-after smaller field of view and avoiding aliasing, or wrap. These techniques hold great promise to broaden the traditional landscape of DTI applications.

Even if the acquired imaging data are as pristine as possible, other limitations with DTI neurography exist and should always be considered. For instance, reversible edema within a nerve may reduce FA enough to halt tractography algorithms through the troubled region. This may not implicate definitive axonal transection, but simply highlights the nature of the technique, which is based on diffusion. A swollen edematous nerve implies potentially more diffusion in general, less regimented pathways for diffusion, more avenues for water molecules to wander, and lower FA. The same is true of, and often encountered in, DTI in the brain. Regardless, halted/truncated fiber bundles during tractography of high-quality data may implicate, localize, and/or confirm a segment of suspected nerve abnormality, despite the fact that the ultimate etiology may be initially nonspecific.

Hemorrhage and surgical clips might also impose magnetic field perturbations that can obscure and alter the apparent diffusion profile near a nerve. These should be kept in mind as alternatives for missing or truncated fibers during tractography attempts.

A good method to adopt is identification of a healthy nerve of similar size in the same imaging volume, which can serve as an internal control for tractography. This may vet the integrity data set and help tune tractography settings before conclusions are drawn.

The future will probably bring forward commercialized methods for rigorous preprocessing of DTI raw data (motion and eddy current corrections, echo planar image distortions, and image registration), quality assurance information, more sophisticated modules for tractography and quantitation of FA, diffusivity, and other metrics.

Diffusion tensor imaging and DT tractography are perfectly poised to introduce a novel, functional dimension of information to conventional MRI sequences and ultrasound in the work-up and follow-up of nerve injuries. 
We must remember, however, that DTI and tractography are ultimately based on mathematical models and assumptions, and require substantial user input, time, and, above all, high-integrity source imaging data. Careful scrutiny of the source data and consistency of imaging approaches over time will be vital to accurate interpretation and follow-up.

\section{References}

1. Chhabra A, Andreisek G, Soldatos T, Wang KC, Flammang AJ, Belzberg AJ, et al: MR neurography: past, present, and future. AJR Am J Roentgenol 197:583-591, 2011

2. Dailey AT, Tsuruda JS, Filler AG, Maravilla KR, Goodkin R, Kliot M: Magnetic resonance neurography of peripheral nerve degeneration and regeneration. Lancet 350:1221-1222, 1997

3. Gunreben G, Bogdahn U: Real-time sonography of acute and chronic muscle denervation. Muscle Nerve 14:654-664, 1991

4. Jones DK: Studying connections in the living human brain with diffusion MRI. Cortex 44:936-952, 2008

5. Kline DG, Hackett ER: Reappraisal of timing for exploration of civilian peripheral nerve injuries. Surgery 78:54-65, 1975

6. Le Bihan D, Poupon C, Amadon A, Lethimonnier F: Artifacts and pitfalls in diffusion MRI. J Magn Reson Imaging 24:478-488, 2006

7. Robert EG, Happel LT, Kline DG: Intraoperative nerve action potential recordings: technical considerations, problems, and pitfalls. Neurosurgery 65 (4 Suppl):A97-A104, 2009

8. Simon NG, Cage T, Narvid J, Noss R, Chin C, Kliot M: Highresolution ultrasonography and diffusion tensor tractography map normal nerve fascicles in relation to schwannoma tissue prior to resection. J Neurosurg 120:1113-1117, 2014

9. Simon NG, Kliot M: Diffusion weighted MRI and tractography for evaluating peripheral nerve degeneration and regeneration. Neural Regen Res 9:2122-2124, 2014

10. Simon NG, Lomen-Hoerth C, Kiernan MC: Patterns of clinical and electrodiagnostic abnormalities in early amyotrophic lateral sclerosis. Muscle Nerve 50:894-899, 2014

11. Simon NG, Narvid J, Cage T, Banerjee S, Ralph JW, Engstrom JW, et al: Visualizing axon regeneration after peripheral nerve injury with magnetic resonance tractography. Neurology 83:1382-1384, 2014
12. Simon NG, Ralph JW, Lomen-Hoerth C, Poncelet AN, Vucic S, Kiernan MC, et al: Quantitative ultrasound of denervated hand muscles. Muscle Nerve [epub ahead of print], 2014

13. Simon NG, Spinner RJ, Kline DG, Kliot M: Advances in the neurological and neurosurgical management of peripheral nerve trauma. J Neurol Neurosurg Psychiatry [epub ahead of print], 2015

14. Takagi T, Nakamura M, Yamada M, Hikishima K, Momoshima S, Fujiyoshi K, et al: Visualization of peripheral nerve degeneration and regeneration: monitoring with diffusion tensor tractography. Neuroimage 44:884-892, 2009

15. West GA, Haynor DR, Goodkin R, Tsuruda JS, Bronstein $\mathrm{AD}, \mathrm{Kraft} \mathrm{G}$, et al: Magnetic resonance imaging signal changes in denervated muscles after peripheral nerve injury. Neurosurgery 35:1077-1085, discussion 1085-1086, 1994

16. Yamasaki T, Fujiwara H, Oda R, Mikami Y, Ikeda T, Nagae $\mathrm{M}$, et al: In vivo evaluation of rabbit sciatic nerve regeneration with diffusion tensor imaging (DTI): correlations with histology and behavior. Magn Reson Imaging 33:95-101, 2015

17. Yeh FC, Verstynen TD, Wang Y, Fernández-Miranda JC, Tseng WYI: Deterministic diffusion fiber tracking improved by quantitative anisotropy. PLoS One 8:e80713, 2013

\section{Disclosure}

The authors report no conflict of interest concerning the materials or methods used in this study or the findings specified in this paper.

\section{Author Contributions}

Conception and design: all authors. Acquisition of data: all authors. Analysis and interpretation of data: all authors. Drafting the article: Gallagher, Simon. Critically revising the article: all authors. Reviewed submitted version of manuscript: all authors. Approved the final version of the manuscript on behalf of all authors: Kliot. Study supervision: Kliot.

\section{Correspondence}

Michel Kliot, Northwestern University Feinberg School of Medicine, NMH/Arkes Family Pavilion, Ste. 2210, 676 N. Saint Clair, Chicago, IL 60611. email: michelkliot@gmail.com. 